Archived version from NCDOCKS Institutional Repository http://libres.uncg.edu/ir/asu/

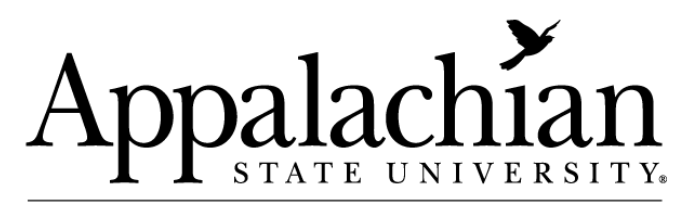

$\overline{\text { B O O N E, NOR T H C A R O L I N A }}$

\title{
Nervous System Excitability And Joint Stiffness Following Short-Term Dynamic Ankle Immobilization
}

\author{
By: Alyssa M. Stirling, Jeffrey M. McBride, Edward K. Merritt, and Alan R. Needle
}

\begin{abstract}
Joint immobilization has been demonstrated to modify neural excitability in subsets of healthy populations, leading to disinhibition of cortical and reflexive pathways. However, these findings may have limited clinical application as most models have investigated casting and rigid immobilization, while many musculoskeletal injuries often utilize dynamic immobilization devices such as boot immobilizers and pneumatic splints that allow for modified ambulation. We therefore aimed to determine the short-term effects of ambulation in ankle immobilization devices on nervous system excitability and stiffness in able-bodied individuals. A repeatedmeasures design was implemented where 12 healthy individuals were tested for cortical excitability to the ankle musculature using transcranial magnetic stimulation, reflexive excitability using the Hoffmann reflex, and ankle joint stiffness using arthrometry before and after $30 \mathrm{~min}$ of ambulation with a boot immobilizer, pneumatic leg splint, or barefoot. Motor evoked potential (MEP), cortical silent period (CSP), Hmax to Mmax ratio, and ankle joint displacement were extracted as dependent variables. Results indicated that despite the novel motor demands of walking in immobilization devices, no significant changes in cortical excitability $(F \geq 0.335$, $P \geq 0.169$ ), reflexive excitability ( $F \geq 0.027, P \geq 0.083)$, or joint stiffness $(F \geq 0.558, P \geq 0.169$ ) occurred. These findings indicate that short-term ambulation in dynamic immobilization devices does not modify neural excitability despite forced constraints on the sensorimotor system. We may therefore conclude that modifications to neural excitability in previous immobilization models are mediated by long-term nervous system plasticity rather than acute mechanisms, and there appear to be no robust changes in corticomotor or spinal excitability acutely posed by ambulation with immobilization devices.
\end{abstract}

Alyssa M. Stirling, Jeffrey M. McBride, Edward K. Merritt, Alan R. Needle (2018). Nervous system excitability and joint stiffness following short-term dynamic ankle immobilization, Gait \& Posture, Volume 59, 2018, Pages 46-52. https://doi.org/10.1016/i.gaitpost.2017.09.028. Publisher version of record available at: https://www.sciencedirect.com/science/article/pii/S0966636217309335?via\%3Dihub 


\title{
Nervous system excitability and joint stiffness following short-term dynamic ankle immobilization
}

\author{
Alyssa M. Stirling, Jeffrey M. McBride, Edward K. Merritt, Alan R. Needle
}

Keywords:

Corticospinal excitability

Motor learning

Joint loading

Transcranial magnetic stimulation

\begin{abstract}
A B S T R A C T
Joint immobilization has been demonstrated to modify neural excitability in subsets of healthy populations, leading to disinhibition of cortical and reflexive pathways. However, these findings may have limited clinical application as most models have investigated casting and rigid immobilization, while many musculoskeletal injuries often utilize dynamic immobilization devices such as boot immobilizers and pneumatic splints that allow for modified ambulation. We therefore aimed to determine the short-term effects of ambulation in ankle immobilization devices on nervous system excitability and stiffness in able-bodied individuals. A repeated-measures design was implemented where 12 healthy individuals were tested for cortical excitability to the ankle musculature using transcranial magnetic stimulation, reflexive excitability using the Hoffmann reflex, and ankle joint stiffness using arthrometry before and after $30 \mathrm{~min}$ of ambulation with a boot immobilizer, pneumatic leg splint, or barefoot. Motor evoked potential (MEP), cortical silent period (CSP), $\mathrm{H}_{\max }$ to $\mathrm{M}_{\max }$ ratio, and ankle joint displacement were extracted as dependent variables. Results indicated that despite the novel motor demands of walking in immobilization devices, no significant changes in cortical excitability ( $\mathrm{F} \geq 0.335$, $P \geq 0.169$ ), reflexive excitability ( $\mathrm{F} \geq 0.027, P \geq 0.083$ ), or joint stiffness ( $\mathrm{F} \geq 0.558, P \geq 0.169$ ) occurred. These findings indicate that short-term ambulation in dynamic immobilization devices does not modify neural excitability despite forced constraints on the sensorimotor system. We may therefore conclude that modifications to neural excitability in previous immobilization models are mediated by long-term nervous system plasticity rather than acute mechanisms, and there appear to be no robust changes in corticomotor or spinal excitability acutely posed by ambulation with immobilization devices.
\end{abstract}

\section{Introduction}

Potentially maladaptive neuroplasticity is frequently described as a barrier to rehabilitation following musculoskeletal injury, causing constraints on the nervous system that may lead to errors in coordination and increase risk of subsequent injury [1]. Immobilization, a frequently implemented therapeutic technique used to attenuate joint forces and provide protection to prevent further injury and allow for typical healing, has previously been described to modify neural plasticity over long-term periods $[2,3]$. Changes to corticospinal excitability have been described following weeks of cast immobilization, reportedly secondary to disuse and decreases in afferent stimulation [4]. These changes in neural excitability are often tied to alterations in joint and musculotendinous stiffness; yet, questions remain regarding the potential impact of these excitability changes on functional status [2,3]. In models of lower extremity injury dynamic immobilization is often provided in devices designed to attenuate load while still allowing for ambulation and normal activities of daily living [5]. These devices create constraints on gait kinetics; however, the use of such immobilization techniques has been associated with improving long-term function after injury [6]. Novel walking tasks have caused neural excitability modifications over short time periods, leading us to hypothesize that altered somatosensation and modified movement patterns from immobilization devices may similarly lead to short-term neural adaptation [7]. Understanding the effects of ambulation in common lower extremity immobilization devices on nervous system function would serve to provide optimal guidance towards clinicians in their abilities to address potentially maladaptive injury-induced neuroplasticity.

Multiple investigations have explored the use of casting and immobilization on corticospinal excitability, yielding conflicting findings. While decreases in motor excitability have been described secondary to 
muscular disuse [8], increases in excitability have often been described as an adaptation to disrupted peripheral somatosensation $[2,4,9]$. These theories suggest that as immobilization serves to deprive joint mechanoreceptors of normal stress, it will lead to $\gamma$-aminobutyric acid (GABA)-related disinhibition and subsequent increases in corticospinal excitability. Increased excitability has also been tied to changes at the segmental level, whereby amplitude of the Hoffmann reflex increases. These reflexive changes have been attributed to sensitization of the muscle spindle from shortening of intrafusal muscle, as well as a reduction in presynaptic inhibition $[3,10]$. However, these phenomena have often been described between 10 days to 6 weeks of immobilization, making the potential consequences of shorter-term immobilization on nervous system function ambiguous. Furthermore, it remains unknown how continued ambulation using more clinically-relevant forms of immobilization may serve to modify corticospinal and reflexive excitability.

In this context, it may be beneficial to consider immobilization as a novel motor learning task for the lower extremity. Often it is difficult to understand the role of the cortex in the learning and adaptation to novel walking paradigms as many typical movements are regulated by subcortical processes $[7,11]$. For instance, central pattern generators are believed to provide the sensorimotor control needed for ambulation $[12,13]$. In cases where central adaptation is required, it is often described within cerebellar Purkinje cells; however, changes have been additionally described in the primary motor cortex (M1) with the level of change proportionate to the complexity of the task [7]. These alterations have been described in novel walking paradigms, including split belt and random speed walking [7]; however, it is unclear whether ambulation in immobilization devices may serve to demonstrate similar adaptations. This is especially of interest in an immobilized joint that would experience diminished somatosensory input during gait.

In the context of joint injury, immobilization has been tied with positive long-term outcomes, despite generally negative effects to the biomechanical function of the joint (e.g. joint contracture, atrophy, etc.) $[6,14,15]$. The potential explanation for this disconnect may be found in discriminating which of these changes are mediated through neural mechanisms, and which are determined by changes to the mechanical characteristics of structures surrounding the joint. By studying the effects of acute immobilization, we may potentially highlight how dynamic immobilization devices are able to modify neural excitability in the absence of tissue adaptation to disuse at the joint. Therefore, it is the purpose of this investigation to describe the alterations to neural excitability to the lower leg muscles following an acute bout of ambulation with clinically- oriented ankle immobilization devices. We hypothesized an increase in cortical and reflexive excitability would occur following $30 \mathrm{~min}$ of ambulation in immobilization devices when compared to a control condition.

\section{Methods}

\subsection{Experimental design}

This study employed a pre-test post-test design with repeated measures. The independent variables were immobilization device and time. Dependent variables included cortical excitability (motor evoked potential size), cortical inhibition (contralateral cortical silent period), and reflexive excitability (Hoffmann reflex).

\subsection{Participants}

Twelve able-bodied and physically active individuals volunteered for this study $(5 \mathrm{M}, \quad 7 \mathrm{~F} ; \quad 22.5 \pm 1.4 \mathrm{yrs} ; \quad 173.05 \pm 17.5 \mathrm{~cm}$; $71.6 \pm 12.7 \mathrm{~kg}$ ). Participants had no history of ankle sprain, fracture or surgery to the lower leg, or history of any lower extremity injury within 6 months of testing. Participants were also excluded if they did not meet criteria for the safe practice of transcranial magnetic stimulation [16]. This included the presence of metal or electronic implant, history of seizure, concussion within the previous 6 months, pregnancy, or treatment for psychiatric or neurological disorder. Participants also completed the Physical Activity Readiness Questionnaire (PAR-Q) to ensure they were capable of performing physical activity [17]. All participants provided university-approved informed consent (Appalachian State University IRB \#15-0207) prior to participation.

\subsection{Instrumentation}

Cortical excitability and inhibition were assessed with transcranial magnetic stimulation (TMS). A magnetic stimulator with a double conical coil $\left(200^{2}\right.$, Magstim, Wales, UK) was used to administer magnetic pulses over the primary motor cortex. Muscle responses were measured using surface electromyography from the tibialis anterior, peroneus longus, and soleus (Bagnoli-4, Delsys, Inc., Boston, MA). Measures from cortical stimulation were compared to peripheral responses from electrical stimulation provided by a constant current stimulator (DS7AH, Digitimer LTD, Hertfordshire, England) with a bipolar bar stimulating electrode consisting of two $8 \mathrm{~mm}$ steel disk electrodes with $30 \mathrm{~mm}$ spacing (Digitimer LTD, Hertfordshire, England).

Joint stiffness was assessed using an instrumented ankle arthrometer (Blue Bay Research, Milton, FL). This device consists of a load cell connected to an instrumented handle, a footplate, and a shin pad connected by a six degree-of-freedom kinematic linkage system. The arthrometer is configured to assess anteroposterior (AP) displacement and inversion-eversion (IE) rotation, as well as the associated force/ torque required for calculation of joint stiffness [18].

\subsection{Procedures}

Participants reported for a total of 3 testing sessions, with a minimum of 3 days and a maximum of 7 days between each testing session. Each session was scheduled at the same approximate time of day. During the first session, participants provided informed consent and completed questionnaires related to inclusion criteria and demographic information. Following this, and for all 3 test sessions, participants were assessed for cortical and reflexive excitability, and ankle joint stiffness prior to and following $30 \mathrm{~min}$ of ambulation on a treadmill. Ambulation was carried out at $1 \mathrm{~m} / \mathrm{s}$, and on each day participants were asked to wear either a pneumatic leg splint (Air-Stirrup, DJO Global, Vista, CA), a pneumatic ankle boot immobilizer (XP Walker, DJO Global, Vista, CA), or to ambulate barefoot (Fig. 1). The leg that was immobilized (test leg) and order of immobilization were randomly determined. The order of testing was randomized for each subject but consistent across test sessions and pre- and post-testing.

For assessment of cortical excitability, participants were first instrumented with electromyography sensors. The area superior to the motor points of the tibialis anterior, peroneus longus, and soleus muscles of the test leg were palpated, shaved, cleaned with alcohol, and lightly abraded according to standard procedures [19]. Electrodes consisted of single-differential electrodes $(99.99 \% \mathrm{Ag})$ with $10 \mathrm{~mm}$ spacing connected to a pre-amplifier (10VN, $-92 \mathrm{db}$ CMRR) placed on the subject's waistband and wire-telemetered to a nearby data acquisition board. These muscles were selected due to their role in regulating ankle joint stability, and because in models of joint injury, modifications in neural excitability are described most commonly to the lower leg muscles on the injured side $[20,21]$. Electrodes were secured using elastic wraps. Once instrumented, participants were asked to sit in an armchair and a tight-fitting elastic cap was placed on the skull. The vertex of the skull was marked on the cap, and the subject was familiarized to the TMS stimuli by gradually increasing pulses until a contraction was visible in the foot. At this intensity, the coil was moved in a small radius in order to locate the "hotspot", identified as the location of the maximum peak-to-peak motor evoked potential (MEP) from the tibialis anterior. Due to the small spatial representation of the lower leg 


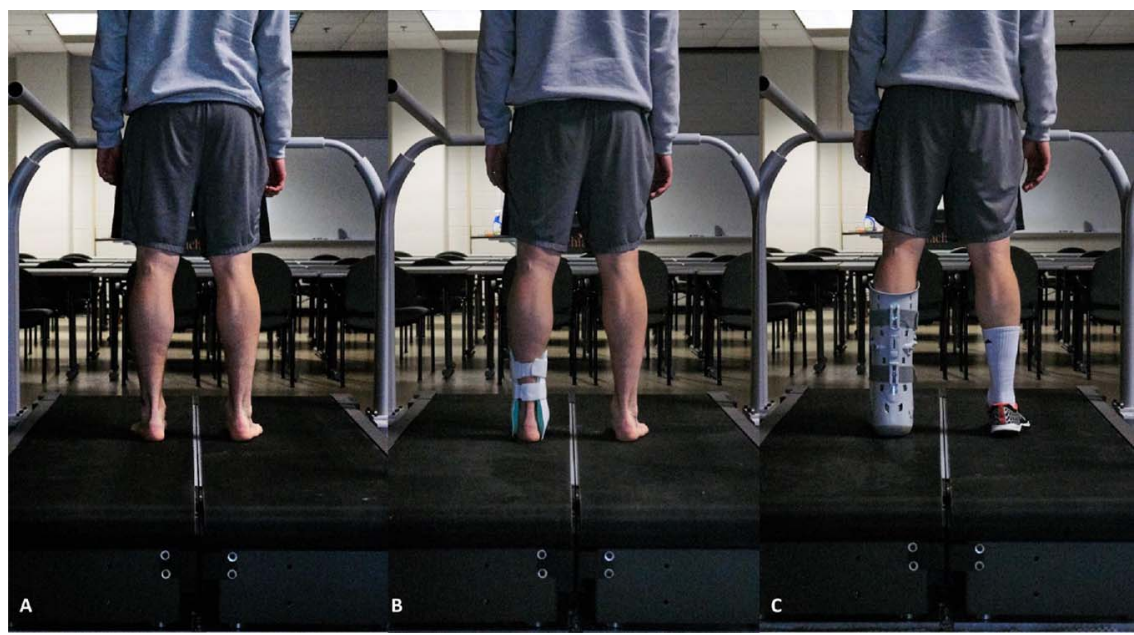

Fig. 1. Immobilization conditions utilized through this study. (a) barefoot condition; (b) pneumatic leg splint condition; (c) ankle boot immobilizer

muscles within the primary motor cortex, and the greater excitability from the tibialis anterior, only the hotspot for this muscle was identified and used throughout testing. At this location, a series of 50 to 60 pulses over a range of intensities from below the point a motor response was observed to above the point a maximal response would be observed were administered over the cortex at $0.2 \mathrm{~Hz}$, as the participant sat relaxed. The peak-to-peak amplitude of the motor evoked potential was plotted against the stimulus intensity to form a stimulus-response curve from which the resting motor threshold (RMT) could be calculated $[22,23]$. Following this determination, subjects were provided visual feedback to maintain a consistent isometric peroneus longus muscle contraction at 15 percent of maximal voluntary isometric contraction as ten pulses at 90 and 110 percent of RMT were applied over the motor cortex in order to obtain active MEPs. Prior to walking each day, the hotspot, RMT, and responses at 90 and 110 percent of RMT were measured, while following walking only responses at 90 and 110 percent of RMT were quantified in order to minimize the time and account for short-term adaptations. EMG data were collected at $2000 \mathrm{~Hz}$.

Reflexive excitability was assessed using the Hoffmann reflex. Participants lay prone as a bar electrode was placed in their popliteal fossa. The location of the sciatic nerve proximal to its bifurcation in tibial and common peroneal divisions was assessed by applying brief pulses and identifying the spot that is able to generate the greatest muscular response across all 3 muscles at the lowest stimulation intensity [24]. Brief electrical pulses (1 ms square pulses) were then applied every $10 \mathrm{~s}$ beginning at 0 and increasing by $2 \mathrm{~mA}$ until a maximal response was observed from all muscles.

For assessment of ankle joint stiffness, the ankle arthrometer was affixed to the ankle by securing the heel and dorsal clamps and fastening the tibial plate to the shin. Five anteroposterior (AP) translations to $130 \mathrm{~N}(50 \mathrm{~N} / \mathrm{s})$ and 5 inversion-eversion (IE) rotations to $4.2 \mathrm{Nm}$ (1 Nm/s) were applied with visual feedback to ensure a consistent rate of loading.

\subsection{Data reduction and analysis}

TMS and Hoffmann reflex data were analyzed in a custom LabVIEW program (National Instruments, Austin, TX). First, responses to electrical stimulation were assessed. Responses in each muscle were partitioned to locate the peak-to-peak values in a time window of $10-40 \mathrm{~ms}$ from the stimulus, and $50-100 \mathrm{~ms}$ from the stimulus. These were used to represent the M-wave (direct response) and $\mathrm{H}$-wave (reflexive response), respectively (Fig. 2). The responses were manually inspected and the maximum $\mathrm{M}$-wave $\left(\mathrm{M}_{\max }\right)$ and maximum $\mathrm{H}$-wave (Hmax) over the range of intensities were extracted, with the Hmax:Mmax ratio used for analysis. Motor evoked potentials from TMS were then manually inspected. The peak-to-peak amplitude from a period of $20-100 \mathrm{~ms}$ following the stimulus were extracted (Fig. 3), and normalized to the $\mathrm{M}_{\max }$ from electrical stimulation. The average active MEP size at each 90 and 110 percent of RMT were used for analysis. Finally, the MEP at 110 percent of RMT for the peroneus longus was assessed for contralateral cortical silent period (CSP), a measured of GABA-related intracortical inhibition. Determination of the CSP followed criteria described by Nilsson et al. in which a series of student's $t$-tests were used to compare logarithmically-transformed EMG activity normalized to pre-stimulus activity to a 4-ms window in order to determine when $t$ tests were no longer significant, indicating EMG activity returned to pre-stimulus levels [25]. Cursors were placed on a plot and a trained investigator confirmed locations were accurate, extracting the time between the start of the MEP and resumption of normal activity as the CSP [26].

The ratio of $H_{\max }$ to $M_{\max }\left(H_{\max }: M_{\max }\right)$ was assessed using two-way analyses of variance (ANOVAs) for each muscle with two within-subjects factors (Device, 3 levels; Time, 2 levels). MEP size was assessed with separate two-way ANOVAs for 90 and 110 percent of RMT within each muscle. Two within-subjects factors were assessed (Device, 3 levels; Time, 2 levels).

Ankle arthrometer data were low-pass filtered $(10 \mathrm{~Hz})$, partitioned from the beginning to end of each load, and manually inspected for consistency across trials in a separate LabVIEW program. Total AP displacement was calculated as the range of maximum to minimum AP displacement. Peak inversion and eversion rotation were measured as the maximum in each respective direction. Arthrometer data were assessed using 2-way analysis of variance (ANOVA) with 2 within-subjects factors (Device, 3 levels; Time, 2 levels). An a priori level of significance was set at 0.05 .

\section{Results}

\subsection{Cortical excitability}

The mean RMT for this subset was $44.167 \pm 5.997$. No significant interaction effects of condition and time were observed for the TA, PL, or SOL at either $90 \%$ RMT (TA: $\mathrm{F}=0.335, P=0.720$; PL: $\mathrm{F}=0.375$, $P=0.693$; SOL: $\mathrm{F}=1.232, P=0.318$ ) or $110 \%$ RMT (TA: $\mathrm{F}=0.345$, $P=0.713$; PL: $\mathrm{F}=0.717, P=0.503$; SOL: $\mathrm{F}=0.998, P=0.390$ ). Additionally no significant main effects of condition were observed for the TA, PL, or SOL at $90 \%$ RMT (TA: $F=1.647, P=0.224$; PL: $\mathrm{F}=1.001, P=0.389$; SOL: $\mathrm{F}=0.781, P=0.475)$ of $110 \%$ RMT (TA: $\mathrm{F}=1.256, P=0.311 ; \mathrm{PL}: \mathrm{F}=1.988, P=0.169 ; \mathrm{SOL}: \mathrm{F}=1.090$, $P=0.360$ ). Furthermore no significant main effects of time were observed for the TA, PL, or SOL at 90\% RMT (TA: $\mathrm{F}=0.708, P=0.424$; PL: $\mathrm{F}=0.680, P=0.434$; SOL: $\mathrm{F}=1.930, P=0.202$ ) of $110 \% \mathrm{RMT}$ (TA: $\mathrm{F}=3.247, P=0.109 ; \mathrm{PL}: \mathrm{F}=0.008, P=0.931$; SOL: $\mathrm{F}=0.282$, 


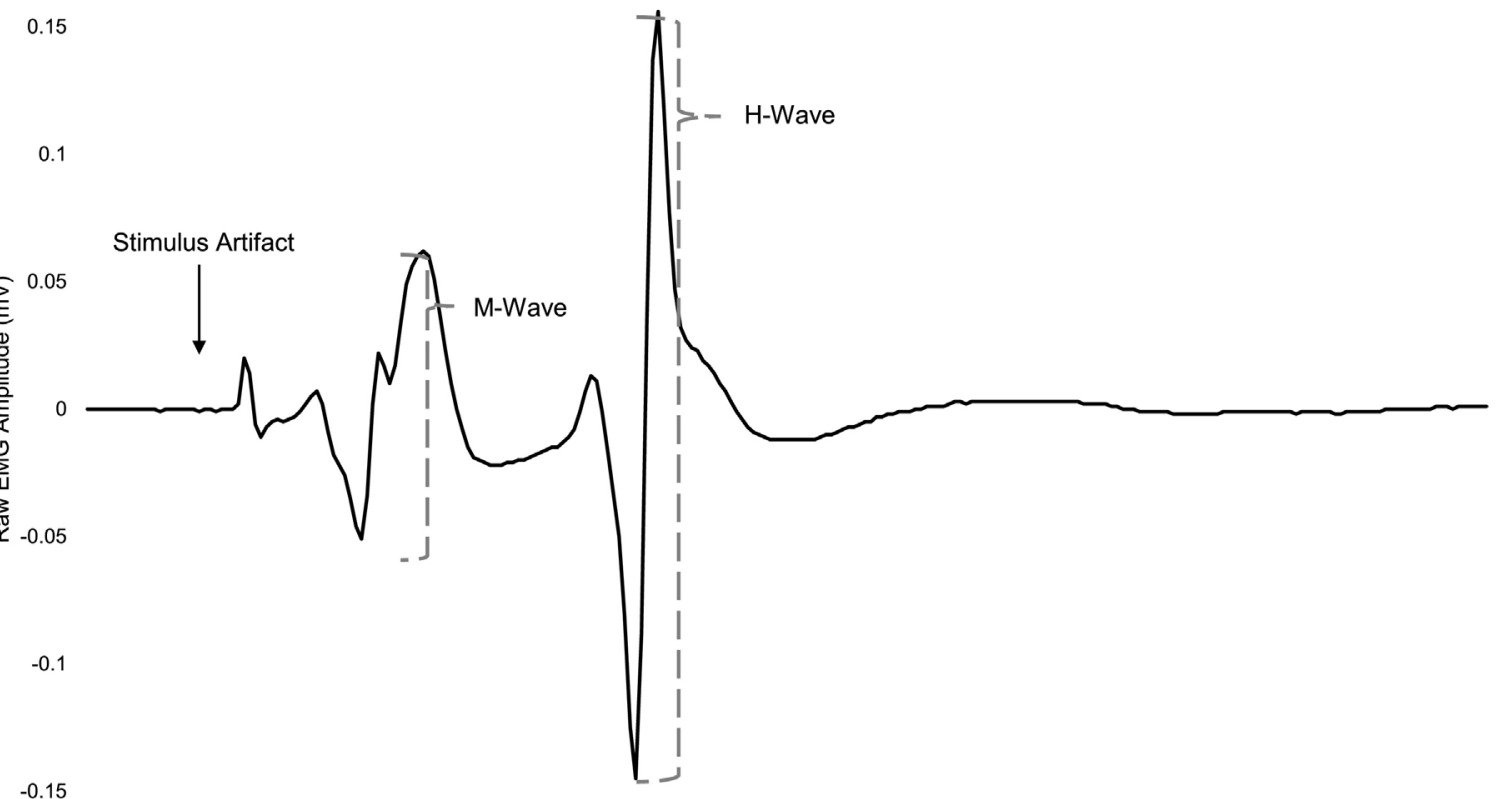

$\mathrm{H}-$ Wave and M-Wave Amplitude over Stimulation Intensities

\section{$\Delta$}

$\Delta$

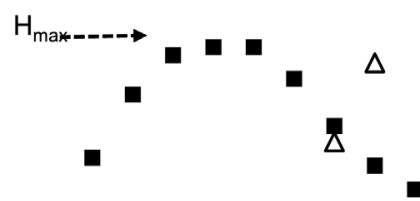

$\triangle$

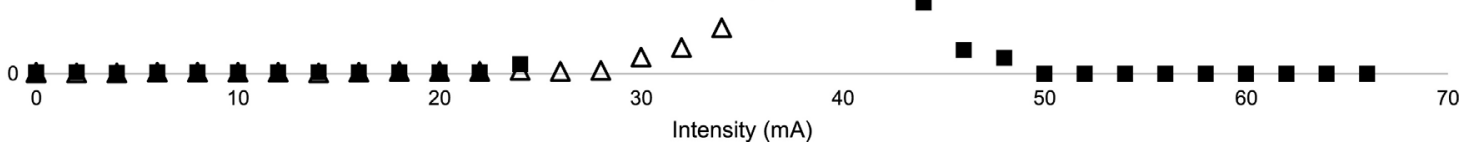

Fig. 2. Sample data from Hoffmann reflex collection. Top: Raw electromyography (EMG) data over $250 \mathrm{~ms}$. Stimulus artifact, direct response (M-wave), and indirect response (H-wave) are labeled. Bottom: Peak-to-peak M-wave and H-wave amplitudes as stimulus intensity increased. Approximate values of maximal H-wave $\left(\mathrm{H}_{\max }\right)$ and maximal $\mathrm{M}$-wave $\left(\mathrm{M}_{\max }\right)$ are labeled. 


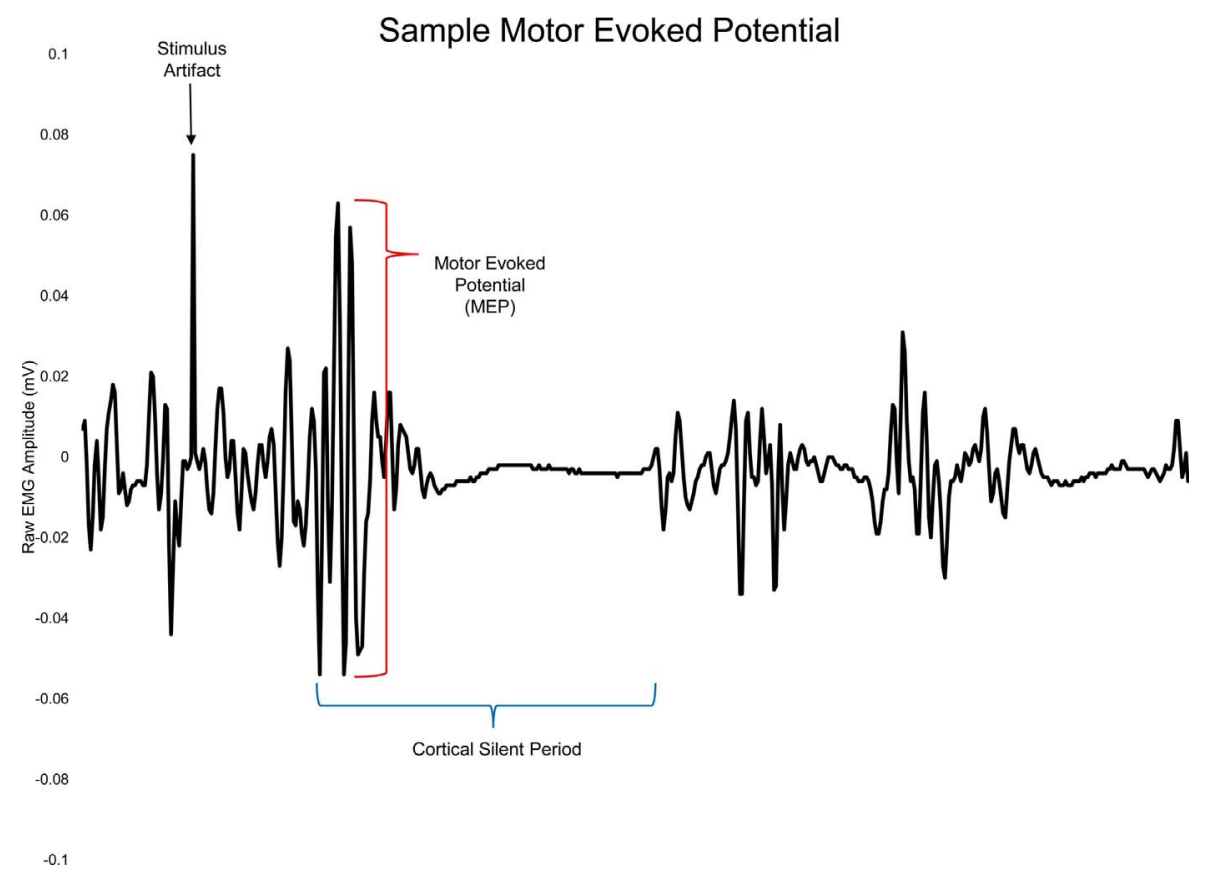

Fig. 3. Sample electromyography (EMG) data from the peroneus longus over a single pulse of transcranial magnetic stimulation in a facilitated (active) condition. The stimulus artifact, motor evoked potential, and cortical silent period are labeled.

Table 1

Cortical excitability (MEP) changes across all muscles, immobilization devices, and time. Abbreviations: RMT, resting motor threshold.

\begin{tabular}{|c|c|c|c|c|c|}
\hline & & \multicolumn{2}{|l|}{$90 \%$ RMT } & \multicolumn{2}{|l|}{$110 \%$ RMT } \\
\hline & & Pre & Post & Pre & Post \\
\hline \multirow[t]{3}{*}{ Tibialis Anterior MEP $\left(\% \mathrm{M}_{\max }\right)$} & Barefoot & $0.086(0.10)$ & $0.071(0.09)$ & $0.191(0.19)$ & $0.157(0.17)$ \\
\hline & Pneumatic Splint & $0.066(0.06)$ & $0.057(0.05)$ & $0.207(0.15)$ & $0.138(0.10)$ \\
\hline & Immobilizer & $0.043(0.05)$ & $0.045(0.05)$ & $0.138(0.13)$ & $0.114(0.11)$ \\
\hline \multirow[t]{3}{*}{ Peroneus Longus MEP $\left(\% \mathrm{M}_{\max }\right)$} & Barefoot & $0.118(0.11)$ & $0.130(0.11)$ & $0.206(0.15)$ & $0.222(0.15)$ \\
\hline & Pneumatic Splint & $0.107(0.22)$ & $0.132(0.07)$ & $0.223(0.09)$ & $0.263(0.17)$ \\
\hline & Immobilizer & $0.090(0.06)$ & $0.087(0.05)$ & $0.168(0.10)$ & $0.160(0.09)$ \\
\hline \multirow[t]{3}{*}{ Soleus MEP (\%M $\left.M_{\max }\right)$} & Barefoot & $0.029(0.06)$ & $0.027(0.05)$ & $0.048(0.06)$ & $0.033(0.06)$ \\
\hline & Pneumatic Splint & $0.017(0.01)$ & $0.023(0.03)$ & $0.055(0.06)$ & $0.057(0.08)$ \\
\hline & Immobilizer & $0.007(0.04)$ & $0.011(0.01)$ & $0.017(0.01)$ & $0.022(0.02)$ \\
\hline \multirow[t]{3}{*}{ Cortical Silent Period (ms) } & Barefoot & & & $282.18(90.2)$ & $316.72(156.3)$ \\
\hline & Pneumatic Splint & & & $271.82(64.3)$ & $296.45(133.5)$ \\
\hline & Immobilizer & & & 310.27 (149.9) & $291.7(132.9)$ \\
\hline
\end{tabular}

$P=0.610)$ (Table 1).

\subsection{Reflexive excitability}

No significant condition by time interaction effect was observed for Hmax:Mmax ratio for the TA $(\mathrm{F}=0.203, P=0.819)$, PL $(\mathrm{F}=0.331$, $P=0.723)$, or SOL $(\mathrm{F}=0.279, P=0.760)($ Table 2$)$. No significant

Table 2

Reflexive excitability (Hoffmann reflex, $\mathrm{H}_{\max }: \mathrm{M}_{\max }$ ) for each muscle before and after each intervention.

\begin{tabular}{llll}
\hline & & Pre & Post \\
\hline Tibialis Anterior & Barefoot & $0.186(0.10)$ & $0.174(0.06)$ \\
& Pneumatic Splint & $0.220(0.06)$ & $0.217(0.07)$ \\
& Immobilizer & $0.218(0.07)$ & $0.190(0.04)$ \\
Peroneus Longus & Barefoot & $0.236(0.17)$ & $0.205(0.13)$ \\
& Pneumatic Splint & $0.222(0.12)$ & $0.212(0.13)$ \\
& Immobilizer & $0.250(0.12)$ & $0.215(0.11)$ \\
& Barefoot & $0.399(0.18)$ & $0.359(0.15)$ \\
& Pneumatic Splint & $0.428(0.19)$ & $0.355(0.15)$ \\
& Immobilizer & $0.412(0.38)$ & $0.375(0.16)$ \\
\hline
\end{tabular}

main effects of condition were observed for the TA $(F=2.921$, $P=0.083), \quad \mathrm{PL} \quad(\mathrm{F}=0.085, \quad P=0.919), \quad$ or $\quad \mathrm{SOL} \quad(\mathrm{F}=0.027$, $P=0.973)$; nor was a significant main effect of time observed in the TA $(\mathrm{F}=1.763, \quad P=0.221), \quad \mathrm{PL} \quad(\mathrm{F}=1.443, \quad P=0.264), \quad$ or $\quad$ SOL $(\mathrm{F}=2.975, P=0.123)$

\subsection{Joint stiffness}

No significant effects of condition by time were observed for AP displacement $(\mathrm{F}=0.558, \mathrm{p}=0.583)$, inversion rotation $(\mathrm{F}=1.746$, $\mathrm{p}=0.203)$, or eversion rotation $(\mathrm{F}=1.968, \mathrm{p}=0.169)$ (Table 3 ).

\section{Discussion}

This study aimed to assess alterations in neural excitability following 30-min of ambulation in clinically-oriented ankle immobilization devices. While prior research has provided evidence of immobilization-induced neuroplasticity, and neural changes during 30min of novel walking tasks, our results revealed no significant changes in cortical or reflexive excitability, nor intracortical inhibition, following the test protocol. While several explanations for these discrepancies may exist, these data suggest that acute synaptic plasticity is 
Table 3

Ankle joint laxity (anterior displacement, inversion rotation, and eversion rotation) before and after each intervention. Abbreviations: AP, antero-posterior.

\begin{tabular}{|c|c|c|c|c|}
\hline & & $\begin{array}{l}\text { AP Displacement } \\
(\mathrm{mm})\end{array}$ & $\begin{array}{l}\text { Inversion } \\
\text { Rotation }\left({ }^{\circ}\right)\end{array}$ & $\begin{array}{l}\text { Eversion } \\
\text { Rotation }\left({ }^{\circ}\right)\end{array}$ \\
\hline \multirow[t]{2}{*}{ Barefoot } & Pre & 8.008 (2.98) & $27.179(10.25)$ & $10.862(3.17)$ \\
\hline & Post & $8.347(3.14)$ & $27.091(10.20)$ & $10.376(2.59)$ \\
\hline Pneumatic & Pre & 7.177 (2.27) & $25.160(9.93)$ & $10.396(3.57)$ \\
\hline Splint & Post & $8.793(3.33)$ & $27.622(10.09)$ & $11.002(2.51)$ \\
\hline \multirow[t]{2}{*}{ Immobilizer } & Pre & 7.660 (3.16) & $30.202(13.24)$ & $13.299(7.05)$ \\
\hline & Post & $8.282(3.45)$ & $26.079(12.88)$ & $10.508(4.68)$ \\
\hline
\end{tabular}

not responsible for alterations to neural excitability of an immobilized leg.

There are two key differences regarding the approach of the current study and previous investigations into the neural effects of ankle immobilization. First and foremost, this is the first study of neural excitability utilizing common clinical ankle immobilization devices, such as immobilizer boots and pneumatic leg splints. These devices are often preferred by clinicians due to their ability to control optimal loading of the joint by allowing for ambulation $[27,28]$. In the case of boot immobilizers, physiologic motion of the ankle joint is minimized, utilizing a rocker sole to assist braking and propulsive forces, while relying on increased torso, hip, and knee motion to allow the foot to clear during the swing phase of gait [29]. Alternately, pneumatic leg splints, while less commonly used, serve to minimize frontotransverse plane motion, while allowing for sagittal plane motion and therefore minimizing swing phase abnormalities. Given that these devices allow for ambulation while imposing mechanical constraints, they would reasonably force the nervous system to adapt to abnormal gait patterns. In fact, the lack of change to joint stiffness suggests alteration to the mechanical ankle structures did not occur. While normal gait has been described to involve minimal input from M1, the adaptation to abnormal walking patterns has been shown to modify excitability to the primary motor cortex and segmental reflexes $[7,13]$. Cortical excitability, as well as disinhibition of $\mathrm{GABA}_{\mathrm{A}}$-mediated pathways, has been described to increase after 20 min of walking under split-belt conditions (right and left legs set to different speeds), and random-speed conditions (belt speed changing every $3 \mathrm{~s}$ ) [7]. These adaptations are similar to those described in the upper extremity as well as motor learning tasks that are nonspecific to stereotyped movements $[30,31]$.

There are several possible reasons why dynamic immobilization did not induce modifications to neural excitability, despite the constraints placed on normal gait patterns. Although ambulation in immobilization devices would cause biomechanical asymmetries, and these asymmetries may add to complexity of walking, it may not have increased task complexity to a degree that would modify neural excitability. The degree of motor cortex adaptation has been tied to both motor learning and motor task complexity, and it could be considered that ambulation in immobilization devices was not sufficient to modify excitability to the ankle musculature [7,32]. It is however possible that the adaptation to the task in this study occurred proximal to the ankle joint. With the ankle immobilized, increased motion from hip and knee musculature would likely be present throughout the gait cycle. These adaptations were not measured though, as previous lower extremity motor learning studies have described adaptation entirely at the ankle musculature (dorsiflexors) $[7,11]$.

The second key difference between our approach to immobilization and those prior studies is the length of intervention. Most prior investigations have investigated immobilization in disuse models, utilizing casting for 10 to 42 days [2,3]. Interestingly, similar to those investigations into adaptation to motor learning, the majority of studies into immobilization have described increases in motor cortex excitability that have been described as pathway-specific. It has been hypothesized decreased somatosensation secondary to immobilization leads to disinhibition and subsequent increased excitability not only at cortical levels, but also segmentally $[3,4,8]$. Furthermore, these changes have been described to persist beyond the immobilized period, and, more importantly, to affect motor learning and adaptation, even after only hours of immobilization [33]. While there are clearly large differences between weeks of cast immobilization and 30-min of ambulation in ankle immobilization devices, considering these results provide context towards the underlying neurophysiologic processes. Our results may lend support to the hypothesis that altered excitability following immobilization is caused by decreased somatosensation, as boot immobilizers and pneumatic splints would provide increased somatosensation to the ankle joint relative to casts. However, to further confirm this, follow-up investigations should observe changes in dynamic immobilization devices over a much longer period of time. Additionally, although short-term immobilization $(10 \mathrm{~h})$ has been demonstrated to affect long-term potentiation \& depression in motor learning tasks, this was not performed concurrently with immobilization as in this study, but rather in the hours following immobilization.

These results have multiple implications for future research into understanding the role of immobilization, and its clinical use, on modifying neuroplasticity. For instance, while we investigated generally robust variables derived from transcranial magnetic stimulation over the motor cortex, additional stimulation parameters have been used in this line of research, including paired-pulse paradigms (i.e. short- and long- intracortical inhibition, intracortical facilitation), and stimulation over alternate areas of the central nervous system (i.e. cerebellum). Stimulation to the cerebellum has been described as a key source of describing walking adaptations after novel walking tasks [7]. Additionally, as previously stated, it is possible that changes may have occurred proximally or in the contralateral limb. A paucity of evidence exists describing these adaptations both after injury and intervention at different points in the kinetic chain.

\subsection{Clinical implications}

In the context of joint injury research, where recurrent injury has been associated with nervous system adaptation, the present study suggests that walking in immobilization devices for short periods of time has no effects on neural excitability to the ankle joint. However, given reports of decreased motor excitability in patients with chronic ankle sprain, and previous studies describing increased excitability following immobilization, we may hypothesize that the use of controlled immobilization devices, such as boot immobilizers, may be able to protect the joint, while ambulation in these devices may be neither helpful nor harmful for modifying neural excitability in this context. However, the effect of immobilization and neural excitability changes on functional status in individuals with injury remains ambiguous, and therefore, we recommend further research explores the effects of clinical ankle immobilization devices on nervous system function over longer periods of time and among populations of injured individuals.

\section{Acknowledgements}

We would like to acknowledge the National Athletic Trainers' Association Research \& Education Foundation and the Appalachian State University Office of Student Research for providing funding in support of this study.

\section{References}

[1] A.R. Needle, A.S. Lepley, D.R. Grooms, Central nervous system adaptation after ligamentous injury: a summary of theories, evidence, and clinical interpretation, Sports Med. 47 (2017) 1271-1288.

[2] C. Leukel, W. Taube, J. Rittweger, A. Gollhofer, M. Ducos, T. Weber, et al., Changes in corticospinal transmission following 8weeks of ankle joint immobilization, Clin. Neurophysiol. 126 (2015) 131-139. 
[3] J. Lundbye-Jensen, J.B. Nielsen, Central nervous system adaptations following 1 wk of wrist and hand immobilization, J. Appl. Physiol. 105 (2008) 139-151.

[4] D.R. Roberts, R. Ricci, F.W. Funke, P. Ramsey, W. Kelley, J.S. Carroll, et al., Lower limb immobilization is associated with increased corticospinal excitability, Exp. Brain Res. 181 (2007) 213-220.

[5] T.W. Kaminski, J. Hertel, N. Amendola, C.L. Docherty, M.G. Dolan, J.T. Hopkins, et al., National Athletic Trainers' Association position statement: conservative management and prevention of ankle sprains in athletes, J. Athl. Train. 48 (2013) 528-545.

[6] S.E. Lamb, J.L. Marsh, J.L. Hutton, R. Nakash, M.W. Cooke, Mechanical supports for acute, severe ankle sprain: a pragmatic, multicentre, randomised controlled trial, Lancet 373 (2009) 575-581.

[7] G. Jayaram, J.M. Galea, A.J. Bastian, P. Celnik, Human locomotor adaptive learning is proportional to depression of cerebellar excitability, Cereb. Cortex 21 (2011) 1901-1909.

[8] J. Liepert, M. Tegenthoff, J.P. Malin, Changes of cortical motor area size during immobilization, Electroencephalogr. Clin. Neurophysiol. 97 (1995) 382-386.

[9] G. Zanette, M. Tinazzi, C. Bonato, A. di Summa, P. Manganotti, A. Polo, et al. Reversible changes of motor cortical outputs following immobilization of the upper limb, Electroencephalogr. Clin. Neurophysiol. 105 (1997) 269-279.

[10] J. Anderson, M.I. Almeida-Silveira, C. Perot, Reflex and muscular adaptations in rat soleus muscle after hindlimb suspension, J. Exp. Biol. 202 (1999) 2701-2707.

[11] D.S. Reisman, A.J. Bastian, S.M. Morton, Neurophysiologic and rehabilitation insights from the split-belt and other locomotor adaptation paradigms, Phys. Ther. 90 (2010) 187-195.

[12] S. Grillner, Locomotion in vertebrates: central mechanisms and reflex interaction, Physiol. Rev. 55 (1975) 247-304.

[13] J.B. Nielsen, Motoneuronal drive during human walking the segerfalk symposium on principles of spinal cord function, Plasticity Repair. 40 (2002) 192-201.

[14] C.A. Reynolds, G.S. Cummings, P.D. Andrew, L.J. Tillman, The effect of nontraumatic immobilization on ankle dorsiflexion stiffness in rats, J. Orthop. Sports Phys. Ther. 23 (1996) 27-33.

[15] G.M.M.J. Kerkhoffs, B.H. Rowe, W.J.J. Assendelft, K.D. Kelly, P.A.A. Struijs, C.N. van Dijk, Immobilisation for acute ankle sprain, Arch. Orthop. Trauma Surg. 121 (2001) 462-471.

[16] S. Rossi, M. Hallett, P.M. Rossini, A. Pascual-Leone, Safety, ethical considerations, and application guidelines for the use of transcranial magnetic stimulation in clinical practice and research, Clin. Neurophysiol. 120 (2009) 2008-2039.

[17] S. Thomas, J. Reading, R.J. Shephard, Revision of the physical activity readiness questionnaire (PAR-Q), Can. J. Sport Sci. 17 (1992) 338-345.

[18] J.E. Kovaleski, J. Hollis, R.J. Heitman, L.R. Gurchiek, A.W. Pearsall, Assessment of ankle-Subtalar-Joint-Complex laxity using an instrumented ankle arthrometer: an experimental cadaveric investigation, J. Athl. Train. 37 (2002) 467-474.

[19] E.F. Delagi, J. Iazetti, A.O. Perotto, D. Morrison, Anatomical Guide for the
Electromyographyer: The Limbs and Trunk, 5 ed., Charles C. Thomas, LTD, Springfield, IL, USA, 2011.

[20] B.G. Pietrosimone, P.A. Gribble, Chronic ankle instability and corticomotor excitability of the fibularis longus muscle, J. Athl. Train. 47 (2012) 621-626.

[21] A.R. Needle, T.W. Kaminski, J. Baumeister, J.S. Higginson, W.B. Farquhar, C.B. Swanik, The relationship between joint stiffness and muscle activity in unstable ankles and copers, J. Sport Rehabil. 26 (2017) 15-25.

[22] A.R. Needle, J.A. Palmer, T.M. Kesar, S.A. Binder-Macleod, C.B. Swanik, Brain regulation of muscle tone in healthy and functionally unstable ankles, J. Sport Rehabil. 22 (2013) 202-211.

[23] H. Devanne, B.A. Lavoie, C. Capaday, Input-output properties and gain changes in the human corticospinal pathway, Exp. Brain Res. 114 (1997) 329-338.

[24] M.A. Hoffman, R.M. Palmieri, C.D. Ingersoll, Simulatneous Hoffmann reflex measurements in multiple muscles around the ankle, Int. J. Neurosci. 113 (2003) 39-46.

[25] J. Nilsson, M. Panizza, P. Arieti, Computer-aided determination of the silent period, J. Clin. Neurophysiol. 14 (1997) 136-143.

[26] T.J. Kimberley, M.R. Borich, K.D. Prochaska, S.L. Mundfrom, A.E. Perkins, J.M. Poepping, Establishing the definition and inter-rater reliability of cortical silent period calculation in subjects with focal hand dystonia and healthy controls, Neurosci. Lett. 464 (2009) 84-87.

[27] J. Ardevol, I. Bolibar, V. Belda, S. Argilaga, Treatment of complete rupture of the lateral ligaments of the ankle: a randomized clinical trial comparing cast immobilization with functional treatment, Knee Surg. Sports Traumatol. Arthrosc. 10 (2002) 371-377.

[28] B.J. McCriskin, K.L. Cameron, J.D. Orr, B.R. Waterman, Management and prevention of acute and chronic lateral ankle instability in athletic patient populations, World J. Orthop. 6 (2015) 161-171.

[29] A. Nepomuceno, M.J. Major, R. Stine, S. Gard, Effect of foot and ankle immobilization on able-bodied gait as a model to increase understanding about bilateral transtibial amputee gait, Prosthet. Orthot. Int. (2017) 1-8 (309364617698521).

[30] M. Tinazzi, G. Zanette, Modulation of ipsilateral motor cortex in man during unimanual finger movements of different complexities, Neurosci. Lett. 244 (1998) 121-124.

[31] C. Sampaio-Baptista, N. Filippini, C.J. Stagg, J. Near, J. Scholz, H. Johansen-Berg, Changes in functional connectivity and GABA levels with long-term motor learning, Neuroimage 106 (2015) 15-20.

[32] M.A. Perez, B.K. Lungholt, K. Nyborg, J.B. Nielsen, Motor skill training induces changes in the excitability of the leg cortical area in healthy humans, Exp. Brain Res. 159 (2004) 197-205.

[33] S.J. Hussain, W.G. Darling, K.J. Cole, Recent history of effector use modulates practice-dependent changes in corticospinal excitability but not motor learning, Brain Stimulation. 9 (2016) 584-593. 